\title{
Paul CHAREST
}

Anthropologue, professeur émérite, département d’anthropologie, Université Laval

(2003)

\section{“QUI A PEUR DES INNUS ?}

\section{Réflexions sur les débats au sujet du projet d'entente de principe entre les Innus de Mashteuiatsh, Essipit, Betsiamites et Nutashkuan et les gouvernements du Québec et du Canada.”}

\author{
Un document produit en version numérique par Jean-Marie Tremblay, bénévole, \\ professeur de sociologie au Cégep de Chicoutimi \\ Courriel: jean-marie tremblay@uqac.ca \\ Site web pédagogique : http://www.uqac.ca/jmt-sociologue/ \\ Dans le cadre de: "Les classiques des sciences sociales" \\ Une bibliothèque numérique fondée et dirigée par Jean-Marie Tremblay, \\ professeur de sociologie au Cégep de Chicoutimi \\ Site web: http://classiques.uqac.ca/
}

Une collection développée en collaboration avec la Bibliothèque Paul-Émile-Boulet de l'Université du Québec à Chicoutimi

Site web: http://bibliotheque.uqac.ca/ 


\section{Politique d'utilisation de la bibliothèque des Classiques}

Toute reproduction et rediffusion de nos fichiers est interdite, même avec la mention de leur provenance, sans l'autorisation formelle, écrite, du fondateur des Classiques des sciences sociales, Jean-Marie Tremblay, sociologue.

Les fichier des Classiques des sciences sociales ne peuvent sans autorisation formelle:

- être hébergé (en fichier ou page web, en totalité ou en partie) sur un serveur autre que celui des Classiques.

- servir de base de travail à un autre fichier modifié ensuite par tout autre moyen (couleur, police, mise en page, extraits, support, etc...),

Les fichiers (.html, .doc, .pdf., .rtf, .jpg, .gif) disponibles sur le site Les Classiques des sciences sociales sont la propriété des Classiques des sciences sociales, un organisme à but non lucratif composé exclusivement de bénévoles.

Ils sont disponibles pour une utilisation intellectuelle et personnelle et, en aucun cas, commerciale. Toute utilisation à des fins commerciales des fichiers sur ce site est strictement interdite et toute rediffusion est également strictement interdite.

L'accès à notre travail est libre et gratuit à tous les utilisateurs. C'est notre mission.

Jean-Marie Tremblay, sociologue

Fondateur et Président-directeur général, LES CLASSIQUES DES SCIENCES SOCIALES. 
Cette édition électronique a été réalisée par Jean-Marie Tremblay, bénévole, professeur de sociologie au Cégep de Chicoutimi à partir de :

Paul Charest

[Anthropologue, professeur émérite, département d’anthropologie, Université Laval.]

"Qui a peur des Innus ? Réflexions sur les débats au sujet du projet d'entente de principe entre les Innus de Mashteuiatsh, Essipit, Betsiamites et Nutashkuan et les gouvernements du Québec et du Canada.”

Un article publié dans la revue Anthropologie et sociétés, vol., 27, no 2, 2003, pp. 185-206. Québec : département d’anthropologie, Université Laval.

[Autorisation formelle de l'auteur accordée le 13 décembre 2007 de diffuser ce texte dans Les Classiques des sciences sociales.]

\section{Courriels : Paul.Charest@ant.ulaval.ca}

Polices de caractères utilisée :

Pour le texte: Times New Roman, 13 points.

Pour les citations : Times New Roman, 12 points.

Pour les notes de bas de page : Times New Roman, 12 points.

Édition électronique réalisée avec le traitement de textes Microsoft Word 2004 pour Macintosh.

Mise en page sur papier format : LETTRE (US letter), 8.5’’ x 11'’)

Édition numérique réalisée le 21 février 2009 à Chicoutimi, Ville de Saguenay, province de Québec, Canada. 


\section{Paul CHAREST}

\section{Anthropologue, professeur émérite, département d’anthropologie,} Université Laval.

\section{"QUI A PEUR DES INNUS?}

Réflexions sur les débats au sujet du projet d'entente de principe entre les Innus de Mashteuiatsh, Essipit, Betsiamites et Nutashkuan et les gouvernements du Québec et du Canada.”

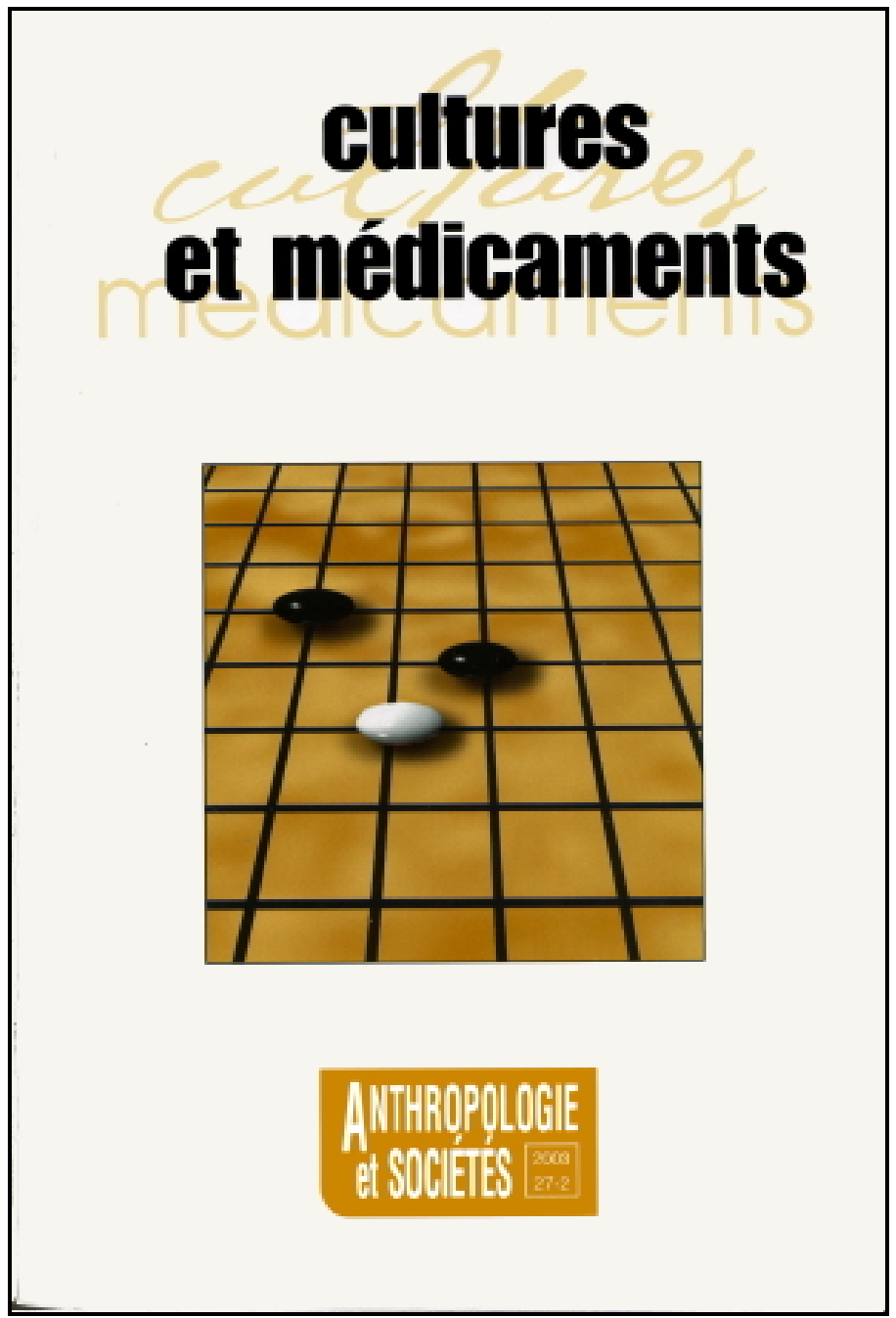

Un article publié dans la revue Anthropologie et sociétés, vol., 27, no 2, 2003, pp. 185-206. Québec : département d’anthropologie, Université Laval. 


\section{Table des matières}

$\underline{\text { Introduction }}$

La politique fédérale de négociations territoriales globales

Petite histoire de la négociation territoriale des Innus

L'entente de principe

Les réactions d'opposition

La participation aux audiences publiques

La diffusion de l'information et la consultation

Les argumentations et les enjeux

Les stéréotypes et préjugés

La « concurrence déloyale»

Le rôle de Guy Chevrette

Les autres moments forts des audiences

La couverture médiatique

Les interventions des parlementaires

Qui a peur des Innus?

$\underline{\text { Références }}$

Figure 1. Superficie du Nitassinan des communautés concernées 
Paul CHAREST

Anthropologue, professeur émérite, département d’anthropologie, Université Laval.

"QUI A PEUR DES INNUS?

Réflexions sur les débats au sujet du projet d'entente de principe entre les Innus de Mashteuiatsh, Essipit, Betsiamites et Nutashkuan et les gouvernements du Québec et du Canada.”

Un article publié dans la revue Anthropologie et sociétés, vol., 27, no 2, 2003, pp. 185-206. Québec : département d’anthropologie, Université Laval.

\section{INTRODUCTION}

\section{$\underline{\text { Retour à la table des matières }}$}

Du 21 janvier au 7 mars 2003, la Commission des Institutions de l'Assemblée nationale du Québec a siégé pendant 12 jours pour discuter des mémoires reçus au sujet du projet d’entente de principe mentionné ci-dessus : 88 mémoires avaient été déposés à l'avance et 72 organismes et personnes se sont présentés devant la Commission pour en débattre. Comme j'ai suivi presque toutes les séances et que j’ai moi-même présenté un mémoire (Charest 2003), j’ai pensé partager avec les lecteurs et lectrices de cette revue mes réflexions personnelles sur ces débats et les enjeux inhérents qui m’apparaissent fondamentaux pour l'avenir des rapports entre les membres allochtones et autochtones de la société québécoise. Mon intention n'est pas de procéder à une analyse systématique des écrits et des discours dont j’ai pris connaissance, mais de présenter une série de sujets ou de thèmes abordés et d’y réagir encore à chaud par des commentaires personnels fondés sur une longue expérience de recherche et de travail de consultant en milieu innu. Mais, d'abord, il m’apparaît nécessaire de fournir au lecteur non familier avec les questions autochtones des informations sur le contexte juridique et politique dans lequel s’inscrit l'actuelle négociation des Innus. 


\section{La politique fédérale de négociations territoriales globales}

$\underline{\text { Retour à la table des matières }}$

La question des droits territoriaux des Autochtones du Canada a une longue histoire parsemée de nombreux jugements de Cour jusqu'en Cour Suprême, qu'il est impossible de résumer en quelques lignes ou paragraphes. Elle a fait l'objet de plusieurs ouvrages et nous y renvoyons donc le lecteur désireux d'approfondir le sujet (Ash 1993 ; Cumming et Mickenberg 1972 ; Kulshinsky 1996 ; Lavoie et al. 1996 ; Long, Boldt et Little Bear 1991 ; Morse 1991). Disons simplement qu'avant les jugements Calder et Malouf de 1973, les droits territoriaux reconnus aux Autochtones se limitaient à des droits d'usufruit temporaires ou résiduels, c'est-à-dire à des droits d'utiliser les terres ancestrales et leurs ressources tant que la Couronne n'en cédait pas la propriété ou l'usage à d'autres pour la colonisation agricole, l'exploitation forestière, la construction de barrages hydroélectriques et de réservoirs, l'établissement de pourvoiries, etc. Jusque-là, les différents traités signés entre la Couronne du Canada et des groupes autochtones comportaient tous une clause d'extinction de tous les droits aborigènes quels qu'ils soient, donc ne les reconnaissaient pas. Cette non-reconnaissance de droits spécifiques aux $\mathrm{Au}-$ tochtones était encore la politique en vigueur du gouvernement du Canada lorsque le jugement Calder fut rendu public. Sans conclure de façon majoritaire à la reconnaissance de droits aborigènes, y compris de droits territoriaux non éteints aux Nisga'as de Colombie-Britannique, ce jugement ouvrait la porte à un éventuel veto des Autochtones sur tout nouveau projet de développement sur les terres non encore cédées par traité. Le gouvernement canadien fit alors une volte-face et proposa la négociation comme moyen de régler les litiges territoriaux issus d'un processus de revendications déjà ancien (les Nishga’as ont amorcé leurs revendications en 1870), mais qu’il avait réussi jusqu'alors à étouffer en modifiant la Loi sur les Indiens pour interdire à des groupes revendicateurs, comme les Nisga'as, de récolter des fonds et de recruter des avocats pour défendre leur cause en Cour. 
Figure 1.

Superficie du Nitassinan des communautés concernées

Retour à la table des matières

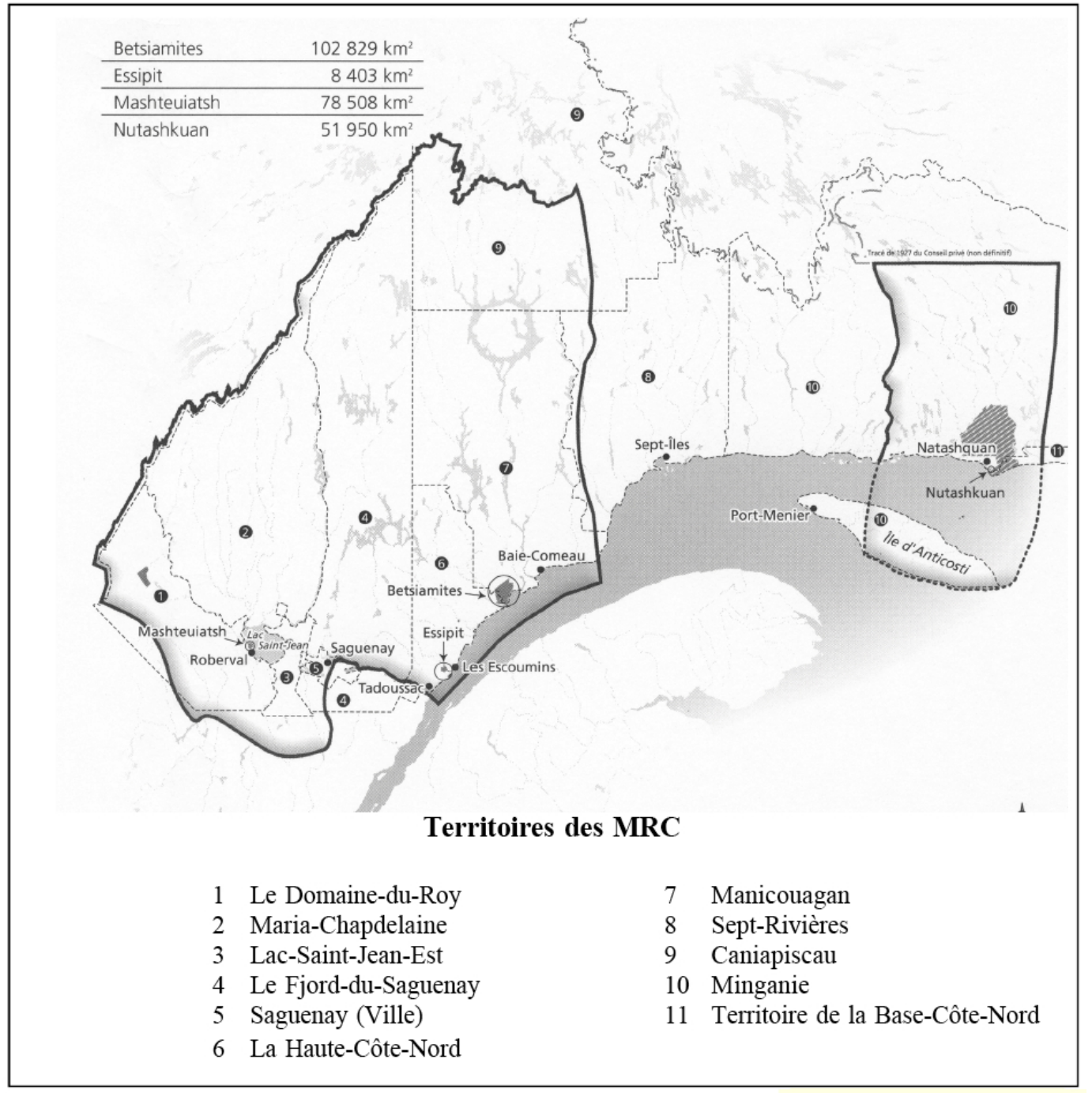

Source : Chevrette 2003

\section{Territoires des MRC}

1. Le Domaine-du-Roy

2. Maria-Chapdelaine

3. Lac-Saint-Jean-Est

4. Le Fjord-du-Saguenay

5. Saguenay (Ville)

6. La Haute-Côte-Nord
7. Manicouagan

8. Sept-Rivières

9. Caniapiscau

10. Minganie

11. Territoire de la Base-Côte-Nord 
Depuis 1973, les Autochtones du Canada qui n’ont pas signé de traité ont donc la possibilité d'entrer dans un processus de négociations territoriales, dites globales, c'est-à-dire sur la base de leurs droits aborigènes non éteints par traité ou par une législation quelconque. Ainsi, à partir de cette date, plusieurs « conventions », ou traités modernes, ont été signées, dont celles avec les Cris, les Inuits et les Naskapis du Nord du Québec en 1975 et 1978, celle avec les Inuits inuvialuit et les Inuits du Nunavut en 1984 et 1991 respectivement, et avec certains groupes dénés des Territoires du Nord-Ouest et du Yukon dans les années 1990. Pendant cette période de négociations intensives impliquant de nombreux groupes autochtones, la nouvelle constitution canadienne adoptée en 1982 reconnaissait l'existence des droits aborigènes sans toutefois les définir. Comme quatre conférences constitutionnelles réunissant tous les premiers ministres des provinces n'y sont pas parvenues non plus, le gouvernement libéral actuel du Canada semble avoir pour politique implicite de laisser les tribunaux en préciser le contenu au fil des jugements successifs, les derniers ayant retenu le plus d'attention étant les jugements Sparrow, Delgamuukw et Marshall qui apparaissent de plus en plus favorables à la reconnaissance de droits territoriaux et de droits d'accès aux ressources pour les Autochtones.

Les Innus du Québec se sont eux-mêmes engagés dans un tel processus de négociation territoriale globale en 1979 en acceptant de se soumettre à la politique fédérale d'alors dont le fondement demeurait l'extinction définitive des droits ancestraux, accompagnée d'autres clauses telles que la reconnaissance de droits de pleine propriété sur des terres de superficies très limitées, de droits prioritaires d'exploitation des ressources fauniques, de compensations monétaires importantes (plusieurs centaines de millions de dollars), d'une participation active au processus d'examen des projets de développement et de protection de l'environnement. En raison des résistances de la part de plusieurs groupes autochtones à sa politique d'extinction des droits territoriaux, le gouvernement fédéral a quelque peu modifié celle-ci en remplaçant le terme " extinction » par celui de "certitude » concernant la propriété future des terres et leur usage (Canada 1987). Toujours insatisfaits de cette clause qui équivalait quand même à l'extinction de leurs droits, les Innus, du moins une partie de ceux-ci, n'ont pas voulu signer une entente basée sur ce principe de certitude. C'est finalement en 2001 qu'un comité de quatre juristes représentant les différentes parties a permis de sortir de l'impasse 
en proposant une solution fondée sur la reconnaissance des droits aborigènes non exclusifs, c'est-à-dire permettant au gouvernement provincial d'exercer aussi sa compétence sur les territoires concernés. Leur proposition est à la base de l'« approche commune » menant à la signature de l'entente de principe dont il sera question dans la suite de ce texte.

\section{Petite histoire de la négociation territoriale des Innus}

$\underline{\text { Retour à la table des matières }}$

Dans la foulée de la Convention de la baie James et du Nord Québécois, était fondé en 1975 le Conseil Attikamek-Montagnais (CAM), dont le mandat principal était de représenter ces deux nations pour leurs revendications territoriales en vertu de la politique fédérale mentionnée plus haut. Personnellement, j'ai été associé au CAM pendant 14 ans, entre 1976 et 1990, comme directeur de recherche et conseiller scientifique à temps partiel, en vertu de prêts de service de la part de l'Université Laval renouvelés annuellement. Dans le cadre de ces fonctions, j'ai participé directement à la préparation de l'énoncé de revendications déposé auprès du ministre des Affaires indiennes et du Nord du Canada en mai 1979 et intitulé Nishastanan Nitasinan (Notre terre nous l'aimons et nous y tenons) (CAM 1979). La conclusion de ce document présente onze principes de négociation qui sont en fait la base d'un projet social pour les deux nations. Ils peuvent être résumés dans la série d'énoncés suivants : la reconnaissance du droit à disposer de soi-même, en tant que peuples autonomes ; la reconnaissance du droit de souveraineté sur les terres ancestrales ; la non-extinction des droits ancestraux ; des dédommagements pour les violations passées de ces droits ; un droit de veto sur tout nouveau projet de développement ; le contrôle sur ces projets et sur les ressources naturelles des territoires de façon à ce que leurs retombées assurent le développement futur des communautés locales ; l'orientation du développement en fonction des valeurs et traditions ancestrales ; l'établissement de rapports égalitaires avec les gouvernements et les membres de la société en général (ibid : 181-182).

Un long processus de négociation s’est amorcé au début des années 1980 et, lors de la dissolution du CAM en 1994, il avait connu quatre négociateurs et quatre structures de négociation différentes. Les négociations ont véritablement 
commencé en 1984 après la nomination de Bernard Cleary comme négociateur en chef, ce qui s'est traduit par la signature en 1986 de deux ententes : une entente cadre, c'est-à-dire un plan de négociation ; une entente sur les mesures provisoires visant à protéger les intérêts des deux groupes amérindiens face aux développements majeurs qui pourraient avoir lieu sur les territoires pendant la période de négociation.

Des mésententes de plus en plus prononcées entre les deux nations membres et aussi à l'intérieur de la nation innue au sujet de l'orientation des négociations et de certains grands principes de base ont conduit à l'éclatement du CAM à la fin de 1994, au moment même où le gouvernement du Québec déposait un projet d'entente de principe (Anonyme 1994).

Après un moment d'arrêt, les négociations ont repris séparément avec trois organisations autochtones qui existaient déjà, soit le Conseil de la Nation Atikamekw, le Conseil tribal Mamuitun (représentant les cinq communautés innues de l'Ouest) et le Conseil tribal Mamit Innuat (représentant les quatre nations innues de l'Est). De son côté, Mamuitun (1996) déposait sa propre proposition d'entente de principe en décembre 1996. Toutefois, deux communautés de Mamuitun (Matimekosh ou Schefferville et Uashat mak Mani-Utenam ou Sept-Îles) se retirèrent du processus de négociation, alors que la communauté de Nutashkuan quitta les rangs de Mamit Innuat pour se joindre aux trois communautés de Mamuitun (Mashteuiatsh, Essipit et Betsiamites). Ces trois communautés avaient déjà avancé la préparation d'une entente de principe fondée sur l' « approche commune » adoptée par les représentants des trois parties à la table de négociation. C'est cette entente de principe qui a été paraphée par les négociateurs au début de 2002 et qui a été rendue publique au mois de mai de la même année. Pour leur part, les trois autres communautés de Mamit Innuat poursuivent actuellement leurs négociations avec les représentants des gouvernements du Québec et du Canada, alors que les communautés de Matimekosh et d'Uashat mak Mani Utenam demeurent toujours à l'extérieur du processus formel de négociation. Cet éclatement de la négociation territoriale des Innus a été présenté comme un problème important par certains intervenants lors des audiences publiques. 


\section{L'entente de principe}

\section{$\underline{\text { Retour à la table des matières }}$}

Le titre complet du projet d'entente issu de l'approche commune est le suivant : Entente de principe d'ordre général entre les Premières Nations de Mamuitun et de Nutashkuan et le gouvernement du Québec et le gouvernement du Canada. J'insiste sur le terme projet, comme l'ont fait plusieurs représentants du gouvernement du Québec, car bien qu'elle ait été paraphée par les trois négociateurs en chef, l'entente de principe n’a pas encore été entérinée par les gouvernements du Québec et du Canada. Par ailleurs, cette entente de principe, relativement peu détaillée à ce stade-ci, continuera à faire l'objet de négociations entre les parties pour en arriver à une entente finale beaucoup plus élaborée, ce qui prendra encore au moins deux ans selon des estimations optimistes, mais probablement beaucoup plus en raison de l'alourdissement du processus expliqué plus loin. Comme les Innus ont entamé leur processus de négociation il y a déjà 23 ans maintenant, « le devoir de résultats », selon l'expression de certains parlementaires, met de plus en plus de pression sur les négociateurs et leurs mandants.

En elle-même l'entente de principe, qui est un texte de quelque 80 pages, présente la base générale de négociation sur laquelle les trois parties se sont entendues par l'adoption de l'approche commune. Les principes généraux à la base de l'entente peuvent être résumés comme ceci :

- la non-extinction des droits aborigènes, ce qui représente une première au Canada ;

- le retrait des communautés innues concernées de la Loi sur les Indiens et du système des réserves ;

- leur remplacement par un gouvernement innu local possédant sa propre constitution et ayant de larges pouvoirs législatifs, exécutifs et judiciaires et par un territoire de pleine propriété appelé Innu Assi ; 
- la conservation des droits de pratique des activités traditionnelles sur l'ensemble des territoires ancestraux appelés Nitassinan et la participation à la mise en valeur de leurs ressources ;

- des compensations monétaires globales totalisant 360 millions de dollars et le versement d'une rente d'au moins $6 \%$ sur les droits payés par les exploitants des ressources naturelles au gouvernement du Québec.

\section{Les réactions d'opposition}

\section{$\underline{\text { Retour à la table des matières }}$}

Comme l'ont rapporté les médias, le projet d'entente a soulevé une tempête de protestations dans certains milieux régionaux, et plus particulièrement à Sept-Îles et à Saguenay, deux localités peu concernées directement. Trois organisations d'opposition ont été rapidement mises sur pied : la fondation Équité territoriale au Saguenay, l'Association pour le droits des Blancs et les Pionniers sept-îliens à Sept-Îles. Elles ont accaparé l'attention des médias électroniques et écrits en véhiculant un ensemble de propos disparates dénaturant les fondements mêmes de l'approche commune. Elles semblent avoir attiré un nombre d'appuis impressionnant dans les deux régions directement touchées par le projet d'entente, soit le Saguenay-Lac-Saint-Jean, ou Sagamie, et la Côte-Nord, comme en témoignent les milliers de cartes de membres vendues par l'Association du droit des Blancs. Les déclarations plutôt incendiaires de l'ancien premier ministre Jacques Parizeau et du député bloquiste Ghislain Lebel contre l'entente ont aussi contribué à échauffer les esprits et à encourager la grogne. À un moment donné, on n'était pas loin d'une paranoïa collective localisée découlant de discours enflammés sur les Indiens qui veulent s'emparer du territoire des Québécois et les en expulser avec la complicité du gouvernement du Québec. Un sentiment d'inquiétude, voire de peur collective a gagné une partie de la population. On pouvait même se croire de retour aux débuts de la colonie et à la grande peur des « sauvages Iroquois ».

Devant cette levée de bouclier toujours aussi localisée, le gouvernement du Québec a organisé, pour présenter les grandes lignes de l'entente, une tournée d'information à laquelle participaient les négociateurs gouvernementaux ou certains de leurs adjoints. J'ai assisté à une de ces séances, celle présentée aux Es- 
coumins en août 2002. Sans être trop enflammées, les réactions de certaines personnes parmi les quelque 200 présentes étaient opposées aux revendications de la communauté locale d'Essipit surtout en ce qui concerne l'élargissement de l'actuelle réserve en un Innu Assi qui s'étendrait sur des terres voisines des municipalités des Escoumins et des Bergeronnes, qui sont par ailleurs des terres de propriété privée encore peu développées au plan résidentiel ou commercial. Les autres inquiétudes concernaient surtout la pratique des activités de chasse, pêche et trappe, la conservation des chalets en forêt et les droits de pêche commerciale en mer. Les mêmes préoccupations ont aussi été formulées ailleurs, dans d'autres localités et sont aussi au centre de bien des mémoires présentés en commission parlementaire.

Le Groupe Cleary et associés, firme de consultants spécialisée en questions autochtones et responsable du site Internet La piste amérindienne, a aussi organisé en octobre et novembre 2002 deux colloques portant sur «l'harmonisation » des rapports entre Autochtones et non-Autochtones, l'un à Chicoutimi, l'autre à BaieComeau. Comme les opposants à l'entente y ont très peu participé, bien qu'ils aient été invités, il n’y a pas eu de réels affrontements entre les deux « camps » et l'harmonie y semblait plutôt de mise. On peut se demander, toutefois, si cette position reflète l'opinion majoritaire des résidents allochtones de la Sagamie et de la Côte-Nord, les opposants faisant beaucoup plus de bruit dans les médias que les partisans de l'entente. Au Saguenay, par exemple, ils monopolisent les lignes ouvertes du poste de radio CKRS et bénéficient de l'appui inconditionnel de son animateur, de même que d'une position éditoriale plutôt favorable du journal Le Quotidien. De plus, le maire de la nouvelle ville fusionnée de Saguenay a fait très ouvertement campagne contre l'entente, utilisant même des fonds publics à cette fin.

Comme ces tournées d'information n'ont pas suffi pour apaiser les esprits, le gouvernement du Québec a décidé de nommer un émissaire spécial, l'ancien ministre Guy Chevrette, pour rencontrer les élites locales et le grand public et faire rapport, puis de convoquer une commission parlementaire pour recevoir toute personne ou tout organisme intéressé et débattre de tous les aspects du projet d'entente de principe. Les séances d'information et de consultation de M. Chevrette ont atteint partiellement l'objectif de calmer le jeu en région, mais les irréductibles, soit essentiellement les membres des trois associations d'opposants 
mentionnées ci-dessus, ont maintenu sans broncher le même discours fondé principalement sur une lecture biaisée du contenu de l'entente et une conception irréaliste des Amérindiens en général et des Innus en particulier sur laquelle nous reviendrons plus loin. Ils ont tout fait pour susciter l'inquiétude sinon la peur de la dépossession au profit des Innus. C'est donc dans cette ambiance de crainte et de suspicion, qui a prévalu pendant tout l'automne dernier, que s'est tenue au début de 2003 la commission parlementaire qui constitue l'objet central de mon propos.

\section{La participation aux audiences publiques}

$\underline{\text { Retour à la table des matières }}$

Les présentations en commission ont été faites par une bonne variété d’organismes : organisations amérindiennes, municipalités, municipalités régionales de comté (MRC), conseils régionaux de développement (CRD), regroupements de chasseurs, de pêcheurs, de trappeurs et de pourvoyeurs, syndicats, partis politiques, représentants d'entreprises forestières et minières. Chez les Autochtones, quatorze organisations et groupes de personnes ont présenté un mémoire devant la commission, y compris les représentants des quatre communautés ayant signé l'entente de principe. Certains organismes innus ont choisi de mettre l'accent sur leurs traditions ancestrales et sur les effets négatifs que les développements industriels ont occasionnés sur leurs territoires de chasse plutôt que sur la discussion de l'entente comme telle. Ils l'ont fait avec une certaine théâtralité en faisant intervenir des aînés en costumes traditionnels et parlant en innu aimum (langue innue). D'autres intervenants amérindiens en ont profité pour souligner leurs propres revendications, comme ce fut le cas pour la Nation huronne-wendat, la Nation atikamekw, la Nation malécite de Viger et la Corporation métisse du Québec. D’autres ont défendu les intérêts d'un groupe de femmes faisant partie de la bande de Mashteuiash ou ceux d'une famille MacKenzie de la communauté innue d’Uashat (Sept-Îles). Il en était de même pour certains non-autochtones intervenant à titre personnel ou comme représentants d'organismes ayant des intérêts bien précis comme le piégeage ou la villégiature. On avait parfois l'impression qu'on ne s’intéressait qu'à des aspects particuliers de l'entente et non 
pas à celle-ci dans son ensemble - plusieurs ne semblaient pas l'avoir lue, encore moins l'avoir bien comprise.

Une vingtaine de personnes ont aussi déposé des mémoires individuels, mais seulement la moitié d'entre elles environ sont venues en débattre devant la commission. Parmi celles-ci, on remarque seulement quatre universitaires : la juriste Andrée Lajoie de l’Université de Montréal ; l’historien Camil Bouchard, de l’UQAC ; le professeur émérite de l’Université Laval, Louis-Edmond Hamelin ; et moi-même. D’autres présentations individuelles ont aussi été faites par de farouches opposants à l'entente, comme Ghislain Lebel et l'historien Russel Bouchard de Chicoutimi. Toutefois, sans en faire un décompte précis, la grande majorité des présentations devant la commission se sont avérées favorables à la conclusion d'une entente, mais plusieurs avec des réserves et des propositions de modifications plus ou moins importantes. De nombreux sujets relevant directement, indirectement et même pas du tout de l'entente ont été abordés. Il serait trop long d'en faire un inventaire complet. Aussi vais-je concentrer mon attention et mes commentaires sur ceux qui m’apparaissent d'un plus grand intérêt.

\section{La diffusion de l'information et la consultation}

\section{$\underline{\text { Retour à la table des matières }}$}

Un commentaire critique, voire une doléance, soulevé par une majorité d'intervenants concerne le manque d'information et de consultation des élites politiques locales, mais surtout de la population en général, sur le projet d'entente. Plusieurs intervenants ont parlé de négociations faites en catimini et gardées volontairement secrètes pour ne pas susciter d'opposition. Chez les opposants à l'entente, les accusations envers le gouvernement du Québec d'avoir joué dans le dos de ses citoyens et de les avoir placés devant un fait accompli ont été particulièrement virulentes. Les représentants politiques, même s’ils ont fait partie d'une table de consultation ont aussi regretté d'avoir été informés trop tardivement ou insuffisamment ou de ne pas avoir été véritablement consultés. Or, comme il a été répété de nombreuses fois tout le long des audiences de la commission, les négociations territoriales des Innus ont été entreprises il y a 23 ans et ont déjà fait l’objet de deux propositions de la part du gouvernement du Québec et d'une de la 
part du conseil tribal Mamuitun. Personnellement, comme j'ai été directement impliqué lorsque j'étais directeur de recherche et conseiller scientifique pour le Conseil des Atikamekw et des Montagnais de 1976 à 1990 et que j'ai suivi d'assez près tout le déroulement des négociations, j'ai accumulé un épais dossier de presse qui démontre qu'assez régulièrement des informations ont été publiées sur un certain nombre d'éléments, tels que les nations et les communautés touchées et leurs effectifs démographiques, les superficies de territoires concernées, les compensations monétaires envisagées, les droits de pratique des activités traditionnelles, les gouvernements innus locaux à mettre en place, etc.

Comment se fait-il alors que les populations régionales concernées n’aient découvert qu'en 2002 la nature des revendications et surtout les grands principes de règlement qui étaient négociés ? Est-ce parce que les propositions précédentes n’avaient pas eu de suite n'ayant pas été acceptées par les trois parties en cause ? Est-ce que la reconnaissance des droits aborigènes, contre laquelle s'est insurgée M. Parizeau, plutôt que leur extinction, selon la politique traditionnelle du gouvernement fédéral, est un nouvel élément qui fait craindre que les Innus ne soient plus désormais soumis aux mêmes lois et règlements que l'ensemble des Québécois ? Ou est-ce que la situation économique, autrefois prospère mais aujourd'hui déclinante du Saguenay-Lac-Saint-Jean et de la Côte-Nord, porte les résidents de ces régions à penser que le gouvernement du Québec les a négligés et qu'il se préoccupe uniquement du bien-être et de l'avenir des Innus? Par ailleurs, le contenu des négociations pouvait-il être rendu public avant même qu'une entente ne soit survenue entre les parties ? Certains ou l'ensemble de ces facteurs ont pu jouer. Quoiqu'il en soit, il semble que l'ensemble de la population des deux régions concernées ait découvert tout d'un coup en 2002 que les Innus étaient en négociations territoriales et que celles-ci avaient abouti à un projet de règlement. Comme il arrive le plus souvent lorsqu'on est a priori contre un projet, les opposants en ont tout de suite profité pour crier au loup en prétextant la noninformation et la non-consultation. Même si cette perception semble assez généralisée et a été reprise en commission parlementaire par les partis d'opposition, jusqu'à quel point ce reproche est-il mérité ? Les trois parties à l'entente et plus particulièrement le gouvernement du Québec ont été pris de court par les réactions fortement médiatisées et ce dernier a essayé de corriger le tir par la tournée d'information des négociateurs provincial et fédéral et par la médiation de Guy 
Chevrette. On a aussi promis en commission parlementaire qu'on procéderait différemment à l'avenir et que la consultation serait désormais assurée par des représentants régionaux à des tables sectorielles, ou même à la table centrale de négociation. Il reste à voir si l'alourdissement du processus de négociation par la multiplication des représentants de toutes sortes d’intérêts (villégiateurs, propriétaires privés, chasseurs, pêcheurs, pourvoyeurs, entreprises forestières et minières, municipalités, etc.) ne viendra pas le ralentir au point de compromettre la conclusion d'une entente finale.

\section{Les argumentations et les enjeux}

\section{$\underline{\text { Retour à la table des matières }}$}

Les principaux arguments apportés en faveur de l'entente, en particulier de la part des négociateurs et des parlementaires ont été les suivants : a) il est préférable de négocier plutôt que d'aller en Cour et de laisser les juges trancher le litige à la suite de longs et coûteux procès ; b) la constitution canadienne de 1982 et la jurisprudence reconnaissent l'existence des droits ancestraux des Autochtones, y compris leurs droits fonciers dans le cas des jugements plus récents et il faut en tenir compte ; c) la future entente mettra fin à l'incertitude actuelle quant aux droits qu'ont ou n'ont pas les Innus, de telle sorte que tout le monde saura à quoi s'en tenir ; d) les retombées de l'entente en termes de développement économique seront profitables pour tous et non seulement pour les Innus ; e) l'entente mettra aussi fin à l'état de dépendance des Innus et leur permettra de prendre en main leurs affaires et de se sortir des conditions sociales difficiles dans laquelle se trouvent les communautés et les familles.

Si la majorité des intervenants a souscrit à ces objectifs et au diagnostic de la situation socio-économique qui lui est associé, une minorité les a rejetés en bloc ou en partie. Ainsi, certains opposants ont même inversé l'argumentation en affirmant que le projet d'entente était inéquitable pour l'ensemble des nonautochtones parce qu'il reconnaissait plus de droits aux Innus, qu’il créait deux classes de citoyens avec des droits inégaux et qu'il contrevenait à la Charte universelle des droits de l'Homme et à la Charte canadienne des droits et libertés. Ce discours inversé sur l’injustice sociale et les iniquités dont seraient victimes les 
Québécois et les habitants des régions concernées, si l'entente était adoptée, peut paraître bien fondé sur des principes placés maintenant au coeur même des «sociétés de droit » - mais essentiellement individuels par opposition aux droits collectifs - comme celles du Canada et du Québec. Mais il est en fait issu d'une fausse conception de l'égalité de tous en droit qui voudrait que tout le monde soit parfaitement égal à tous les points de vue. Il nie de fait l'existence de droits particuliers pour certaines collectivités, y compris les droits des minorités et les droits des Autochtones. La méconnaissance des droits des Autochtones du Canada et même le refus flagrant de leur reconnaissance chez certains a été pour moi sinon une surprise du moins une grande déception lors des audiences. Il en est de même pour l'ignorance du statut juridique particulier dans lequel se trouvent les Amérindiens en vertu de la Loi sur les Indiens et des conséquences de toutes sortes que cela implique en tant que subordination aux décisions du gouvernement fédéral et de ses agents - financement restreint des programmes et services, limitations diverses en ce qui concerne la propriété foncière et immobilière, la capacité d'emprunter, les dispositions testamentaires, etc.

\section{Les stéréotypes et préjugés}

\section{$\underline{\text { Retour à la table des matières }}$}

Ainsi, plusieurs intervenants ont véhiculé les tenaces stéréotypes et préjugés paraissant régulièrement dans les médias selon lesquels les Amérindiens sont grassement entretenus par l'État à même les impôts et les taxes des autres Québécois et Canadiens, alors qu'ils n’en paient aucun, qu’ils ne paient ni loyer ni électricité et qu’ils sont subventionnés pour tout ce qu’ils entreprennent. Les gouvernements leur donneraient tout et iraient même au devant de leurs désirs, malgré le fait qu'ils ne respectent aucune loi ni règlements, en particulier ceux qui concernent la chasse et la pêche et l'exploitation des ressources fauniques en général, contrairement aux bons citoyens non autochtones qui, eux, seraient des modèles en ce domaine. Finalement, certains ont joué les prophètes de malheur en prétendant que l'entente, si elle était signée, serait catastrophique pour les régions, pour les entreprises qui y travaillent et pour l'avenir des jeunes qui n'auraient plus qu'à émigrer à l'extérieur pour y trouver un avenir meilleur. 
Il est difficile de comprendre comment une entente touchant quelque 8000 Innus, leur octroyant un peu plus de terres en pleine propriété et reconnaissant leurs droits de pratiquer des activités traditionnelles sur leurs terres ancestrales puisse être perçue comme risquant d'entraîner des effets aussi terribles pour les 300000 non-Innus habitant les mêmes territoires. Il faut vraiment être motivé par une peur incompréhensible ou par le racisme, accusation que les plus farouches opposants à l'entente récusent vigoureusement. Mais, « quand on veut tuer son chien on dit qu'il a la rage », dit le proverbe ! Ainsi, quand on veut se débarrasser des Amérindiens on prétend qu’ils ne font rien mais qu’ils veulent tout avoir, qu'ils vivent à « nos crochets », ou encore qu'ils n'existent plus comme groupe ayant des droits ancestraux, leurs ancêtres s’étant «métissés » avec des Amérindiens d'autres nations ou, encore pire, avec des « Blancs ».

Telles sont les thèses « disparitionnistes » soutenues par les historiens Russel Bouchard et Nelson-Martin Dawson auxquelles se réfèrent les opposants à l'entente pour la discréditer. Celles-ci ont cependant fait l'objet d'une forte opposition dans les médias écrits, dans Le Devoir de Montréal et Le Soleil de Québec plus particulièrement, de la part de plusieurs anthropologues québécois (Serge Bouchard, Paul Charest, Jean-Pierre Garneau, Josée Mailhot, Rémy Savard, Pierre Trudel). J’ai déjà réfuté dans les pages de cette revue (Charest 1996) la thèse « disparitionniste » de R. Bouchard concernant les Innus du Saguenay-Lac-SaintJean exposée dans son livre Le dernier des Montagnais (1995), mais elle a été reprise avec plus d'ampleur par Dawson (2001) dans une série de rapports commandés par Hydro-Québec et repris de façon biaisée par le journaliste Michel Morin à la télévision de Radio-Canada, au mois d’octobre 2002.

\section{La « concurrence déloyale »}

\section{$\underline{\text { Retour à la table des matières }}$}

Un autre argument avancé par certains opposants à l'entente de principe est celui de la « concurrence déloyale », mais il concerne uniquement la communauté d’Essipit qui serait un cas particulier en raison de sa situation économique prétendument privilégiée comparativement à celle des trois autres communautés associées à l'approche commune. Cette accusation — ou autre plainte — provient de 
personnes et d'organismes de la municipalité des Escoumins dans laquelle Essipit se trouve enclavée, d'un organisme sous-régional comme la MRC Haute-CôteNord et même d'un organisme régional, le CRD-Côte-Nord. Selon certains de leurs représentants, le non-paiement de taxes et d'impôts par les personnes qui ont un statut indien et les subventions que les bandes reçoivent des organismes gouvernementaux feraient que leurs entreprises exercent une «concurrence déloyale » envers les commerçants locaux non autochtones en offrant des prix avantageux, ce qui les obligerait à vendre leurs entreprises que la bande s'empresserait aussitôt d'acheter à bas prix. Comme je fais du terrain depuis cinq ans à Essipit et aux Escoumins, il m’apparaît que ces accusations ne sont guère fondées sur des faits solides. Il est vrai que la bande est nantie économiquement et qu'elle possède plusieurs entreprises, principalement dans le secteur récréotouristique : pourvoiries, excursions aux baleines en bateau et en pneumatiques, centres d'hébergement, campings, centre communautaire avec installations sportives intérieures et extérieures, dépanneur, boutique d'artisanat, station d'essence ; ainsi qu'une entreprise d'aménagement forestier, un bateau de pêche au crabe d'acquisition récente et une radio communautaire diffusant pour toute la HauteCôte-Nord.

Ainsi, en 2001, 117 des quelque 160 emplois permanents ou saisonniers fournis par le conseil de bande étaient reliés directement au développement économique de la communauté. Par contre, les entreprises du conseil de bande payaient plus de 330000 \$ de taxes fédérales et provinciales et autres redevances. Par ailleurs, une majorité des emplois étaient occupés par des non-Indiens qui, eux, paient des taxes et des impôts. De plus, environ 80\% des dépenses des entreprises et du conseil de bande étaient effectuées hors de la réserve, dans les commerces de la municipalité voisine des Escoumins, dans la sous-région ou ailleurs au Québec. La majorité des entreprises de la bande ne concurrencent pas celles de la municipalité voisine, sauf dans le domaine de l'hébergement. La bande possède bien un dépanneur et un bar, mais elle n'a pas d'épicerie et elle ne gère pas elle-même le petit restaurant situé dans la réserve ; elle n’a pas non plus de magasin de vêtements, de magasin de meuble, de garage de vente et de réparation automobile, de lunetterie, de papeterie, de pharmacie, etc., tous commerces que l'on retrouve dans la municipalité des Escoumins. Du côté des prix, les listes comparées que j'ai pu consulter montrent qu'il n’y a presque pas de différence entre ceux 
qu'affiche le dépanneur de la réserve et ceux d'une des épiceries de la municipalité utilisée comme point de comparaison. Il en est de même pour les prix de l'essence. Selon les types d'unités de logements pour touristes, les prix d'Essipit peuvent être soit plus élevés, soit équivalents ou un peu moins élevés que ceux des établissements des Escoumins. Pour ce qui est des tarifs des excursions aux baleines à partir du quai de Bergeronnes, ils sont presque identiques à ceux des concurrents travaillant à l'extérieur de Tadoussac, y compris un entrepreneur privé membre de la bande d’Essipit. Il est vrai que la bande a acheté quelques commerces et autres propriétés dans la municipalité des Escoumins, dont un motelcamping bien situé sur le bord de la mer. Par contre, récemment deux de ses membres ont acheté des commerces (une quincaillerie et une épicerie), mais à titre personnel, et c'est peut-être la succession de ces trois achats rapprochés qui a suscité la réaction de certaines personnes et ces accusations de « concurrence déloyale ». Or, de façon objective, après ce bref tour de la situation, les activités économiques de la bande d'Essipit ont bien plus de retombées positives que de conséquences négatives sur la municipalité voisine.

Les raisons du succès de la bande sont à chercher ailleurs que dans la concurrence déloyale, à savoir dans les facteurs suivants : a) une grande stabilité dans le leadership politique et administratif ; b) des gestionnaires compétents et travaillant de façon concertée ; c) l'approche communautaire qui consiste à réinvestir les gains des entreprises dans le développement de celles-ci ou dans l'acquisition de nouveaux commerces selon une planification rigoureuse à moyen et à plus long terme ; d) l'intéressement des employés cadres et subalternes ; e) la qualité des services offerts par la bande à ses clients, de même que la qualité des services communautaires (logements, loisirs, soins de santé de première ligne, éducation, etc.) offerts aux membres de la bande. Il s'agit là, par contre, d'un modèle qui n’est pas véritablement exportable tel quel dans d'autres communautés innues pour différentes raisons, dont la petite taille des effectifs de la bande, sa proximité d'un important centre touristique, Tadoussac, et sa proximité des grands centres urbains d'où vient en grande partie sa clientèle. À constater les réactions négatives que soulève le succès d'un groupe autochtone en termes de développement économique, on peut se demander si cette "jalousie », comme l'appellent certains, ne vient pas simplement du fait que des Autochtones qui font de bonnes 
affaires et qui s’enrichissent ne peuvent être considérés comme de « vrais Indiens ».

\section{Le rôle de Guy Chevrette}

$\underline{\text { Retour à la table des matières }}$

Comme je l'ai dit précédemment, dans la tourmente de l'opposition et des questionnements au sujet de l'entente de principe, l'ex-ministre Guy Chevrette a été appelé en renfort comme «mandataire spécial du gouvernement du Québec » ou, selon une expression reprise dans plusieurs médias, comme « pompier » avec pour objectif, sinon d'éteindre, du moins de circonscrire le feu au mieux. On peut reconnaître qu'il a pris sa tâche très au sérieux et qu'il s’y est consacré entièrement pendant environ six mois, multipliant les rencontres avec de nombreux groupes et individus de toutes vocations et tendances, ne reculant jamais devant les groupements et assemblées hostiles, comme il a pu en rencontrer à Sept-Îles particulièrement. J'ai eu une rencontre privée d'une heure avec $\mathrm{M}$. Chevrette et je dois dire qu’il a été très réceptif à mes propos, écoutant beaucoup plus qu’il n’a parlé. Si on l'a accusé d'être à la fois juge et partie dans le dossier (il était encore ministre des Affaires autochtones lorsque l'approche commune a été adoptée dans ses grandes lignes), on peut constater que ses prises de position publiques et son rapport ont effectivement contribué à diminuer la tension et à lui attirer des commentaires positifs de la part de nombreuses personnes concernées directement ou indirectement, comme j'ai pu m’en rendre compte personnellement.

Le rapport de «mission » de M. Chevrette, intitulé Pour la négociation d'un traité juste et équitable a été officiellement déposé devant la Commission des Institutions lors de la première journée des audiences publiques, le 21 janvier 2003. Il comprend une cinquantaine de pages et se divise en quatre grandes parties : 1) «Les grands constats »; 2) «Les territoires en cause et les principales modalités qui s’y appliquent » ; 3) « Le processus de négociation et autres considérations »; 4) et des « recommandations ». Il n’est pas de mon propos d'en faire un résumé et je vais seulement souligner quelques points du rapport qui m’apparaissent particulièrement importants. Ainsi, dans la conclusion de la partie sur « les constats » M. Chevrette écrit ceci : 
En conséquence, le processus de négociation doit être repensé de manière à y favoriser une participation accrue des populations régionales. Il s’agit d'une condition incontournable au succès des négociations en cours.

Chevrette $2003: 7$

Dans la partie sur « Les territoires en cause... », le rapport remet en question l'inclusion de l'île d'Anticosti et de la partie « dite sud ouest » (de la rivière Saguenay à Portneuf) dans les Nitassinan respectifs des communautés de Nutashkuan et de Mashteuiatsh. De plus, il rappelle que les « paramètres » du gouvernement du Québec « établissent que l’intégrité du territoire du Québec ne peut être compromise » (ibid. : 17). Ils souligne aussi que : "Avec la création des Innus Assi, les Innus échappent à la dépendance dans laquelle ils sont maintenus par la Loi sur les Indiens et ils accèdent à la responsabilisation » (ibid.). Il constate, par ailleurs, que « La situation d'Essipit, des Escoumins et des Bergeronnes est plus complexe » en raison d'une dispute au sujet de la délimitation de l'Innu Assi et qu’il y existerait « une détérioration du climat entre les communautés en présence et dont on s'accuse mutuellement » (ibid. : 19).

Dans la partie du rapport sur le «processus de négociation », M. Chevrette propose une restructuration permettant à des représentants des deux régions concernées de participer à des comités directeurs régionaux de négociation et à des tables sectorielles de consultation (ibid. : 21). Il admet que ces mesures alourdiront le processus de négociation, mais « qu’il s’agit là du prix à payer non seulement pour en arriver à une entente équitable pour tous, mais également pour lui assurer la nécessaire acceptabilité sociale qui lui fait passablement défaut actuellement » (ibid. : 22). Comme les recommandations contenues dans le rapport reprennent les principaux éléments que je viens de rapporter, il n'est pas utile de s’y attarder, sauf pour mentionner que la septième recommandation veut interdire la pratique des activités traditionnelles «à l'intérieur des périmètres de territoire ayant un statut particulier » tels que les réserves écologiques, les habitats fauniques et sanctuaires, les zones urbanisées des municipalités, les emplacements de villégiature privée, etc. (ibid. : 27). 
Parce qu'il fait une large place à beaucoup de doléances des gens des régions qu'il a rencontrés, le rapport de $\mathrm{M}$. Chevrette a été dans l'ensemble très bien reçu par ceux-ci, par plusieurs intervenants devant la Commission et par les parlementaires. Il ne l'a pas été par les irréductibles opposants parce qu'il fermait la porte à la tenue de référendums ou à un moratoire sur la négociation que ceux-ci réclament à cor et à cri. En effet, comment peut-on penser soumettre les droits d'une minorité autochtone, qui sont déjà reconnus de toute façon par la constitution canadienne, au diktat de la majorité non autochtone ? De leur côté, les leaders des communautés innues concernées ont trouvé que les propositions du mandataire affecteraient passablement à la baisse leur projet d'entente, mais ils s'y sont résignés dans le but de la rendre plus « socialement acceptable ». Il s'agit là de nouvelles concessions de leur part, qui s'ajoutent à bien d'autres comme je l'ai démontré dans mon mémoire (Charest 2003).

\section{Les autres moments forts des audiences}

$\underline{\text { Retour à la table des matières }}$

Si la présentation du rapport de $\mathrm{M}$. Chevrette, qui était très attendue, a constitué un des moments forts des audiences publiques, il y en a eu bien d'autres qui m’ont particulièrement touché. Je me limiterai à en commenter quelques-unes. Du côté des entités politiques et administratives, je voudrais d'abord souligner la présentation du maire de la ville de Roberval, ma ville natale, que j'ai trouvé d'une grande qualité humaine par sa référence à Roberval et Mashteuiatsh comme « un vieux couple » et à la volonté de poursuivre et d'améliorer, à travers l'entente, une cohabitation déjà considérée comme harmonieuse. J'ai aussi été impressionné par les prises de positions positives des syndicats et plus particulièrement par celle de la Centrale des syndicats du Québec lors de la présentation de son mémoire le 11 février. L'intitulé de son mémoire dit tout sur son appui au projet d'entente : «Pour en finir avec la tutelle et le sous-développement : l'autonomie gouvernementale aux Premières Nations ». Parmi les mémoires individuels, celui du Dr Stanley Vollant, premier et seul médecin d'origine innu, m’a beaucoup touché de même que de nombreuses personnes présentes et les parlementaires membres de la commission. À l'aide de nombreuses données, le Dr Vollant a démontré sans 
fard l'état de détresse sanitaire et sociale dans laquelle sont plongées la plupart des communautés innues, y compris celle dont il est originaire, Betsiamites. Par ailleurs, la réelle volonté de partenariat d'affaires de la part des représentants souvent de très haut « gradés » - des entreprises forestières et minières, m’a quelque peu surpris, car je m'attendais au contraire à beaucoup plus de résistance de leur part. En ce qui concerne l'exploitation des ressources naturelles, cette résistance est davantage venue des nombreuses associations de chasseurs, pêcheurs et trappeurs qui voient dans le projet d'entente de nombreux sujets d'inquiétude pour la poursuite de leurs activités préférées en forêt et qui ont fait souvent de nombreux commentaires et recommandations de détails sur des aspects ponctuels de l'entente qui ne sont même pas inclus actuellement dans le document dit de "portée générale », mais qui devront être précisés dans des ententes sectorielles. Autre présentation notable, celle de notre collègue ethnolinguiste Josée Mailhot, qui a démoli avec brio les prétentions de certains historiens régionaux selon lesquelles la langue parlée par les Innus de Mashteuiatsh ne serait par la langue innue.

J'ai moins apprécié les présentations de certains irréductibles opposants à l'entente, en particulier celles du maire Jean Tremblay de Saguenay et de Russel Bouchard. Ne reculant pas devant le ridicule, ils ont affirmé, respectivement, que la ville de Saguenay était la plus pauvre au Canada et que les Canadiens français devaient être considérés comme des Autochtones au sens de l'article 35(2) de l'Acte constitutionnel de 1982. Il est malheureux, et en bonne partie incompréhensible, que de tels leaders d'opinion aient un aussi vaste auditoire dans la région du Saguenay, tellement leurs idées sont saugrenues, non fondées, empreintes d'idées revanchardes contre les Innus, et à la limite du racisme, puisque selon R. Bouchard la « race montagnaise » n'existe plus. Heureusement qu'il y a d'autres maires comme celui de Roberval pour voir dans les Innus des partenaires plutôt que des ennemis et des historiens comme Camil Girard pour rappeler la première alliance établie en 1603 à l'embouchure du Saguenay entre Champlain et Du Pont Gravé comme représentant de la France et les représentants de trois nations amérindiennes, dont celle des Montagnais. Une autre affirmation grotesque que j'ai relevée en tant qu'anthropologue est celle du président que la Corporation métisse du Québec affirmant que le terme «autochtone» ne devait s'appliquer qu'en Afrique, berceau de l'Humanité, et que le terme « indigène » qu'il utilisait exclu- 
sivement est celui qui devait prévaloir en Amérique du Nord et au Canada. Finalement, une déception a été le forfait des Cris qui n’ont pas fait la présentation publique de leur mémoire prévue pour la séance du 6 mars. Selon moi, il aurait été fort intéressant que les Cris fassent bénéficier publiquement les Innus de leur expérience de négociations et des difficultés découlant de la mise en oeuvre de leur entente, car il est probable que les Innus se heurteront aux mêmes problèmes.

\section{La couverture médiatique}

\section{$\underline{\text { Retour à la table des matières }}$}

Les comptes rendus médiatiques des débats de la commission parlementaire ont été très décevants. À part le premier jour où les caméras étaient présentes, la télévision s’est limitée à couvrir la visite de certains ténors parmi les opposants, habitude de sensationnalisme propre à ce type de journalisme. Les journaux « nationaux » francophones comme La Presse et Le Devoir n’ont à peu près pas évoqué l'évènement. Selon moi, ils sont passés à côté d'un débat de société majeur qui n’a pas fini d’avoir des répercussions dans l'avenir, non seulement pour les régions directement concernées mais pour l'ensemble du Québec. En effet, le dossier de la négociation avec les Innus est loin d’être clos, car la négociation avec les quatre communautés ayant adopté l'approche commune dureront encore des années ; de plus, les négociations avec les cinq autres déjà en cours ou souhaitées prolongeront le processus, d'autant plus que le gouvernement du Québec veut parvenir à une entente avec la totalité des Innus du Québec. Cet objectif m’apparaît irréaliste d'après ce que je connais des positions de Matimekosh et des Uashat mak Mani Utenam par rapport aux négociations territoriales. Par ailleurs, selon la politique fédérale, rien n’oblige à ce que les ententes soient signées seulement avec des nations autochtones au complet, comme ce fut le cas pour la Convention de la baie James. De nombreux exemples dans les Territoires du Nord-Ouest, le Yukon et la Colombie-Britannique le démontrent. Par contre, il est vrai que le fait de négocier séparément plusieurs ententes avec des communautés de la même nation ou même de nations voisines pose de réels problèmes de chevauchements des territoires, puisque dans le passé ceux-ci n’avaient pas de réelles 
frontières, les différents groupes ou bandes locales et régionales exploitant en commun ou en alternance des zones limitrophes.

Enfin, d'autres groupes amérindiens occupent aussi des régions où la majorité de la population est non autochtone (les Atikamekw qui sont aussi depuis longtemps engagés dans un processus de négociation, ou comme les Algonquins qui s'y engageront tôt ou tard), et ils en viendront aussi à des projets d'entente qui risquent de soulever à leur tour de vives réactions chez les allochtones des régions concernées. Si l'entente avec les Cris a suscité tant de controverses et de disputes, ce n'est rien à côté de ce qui nous attend dans le futur au fur et à mesure que les autres nations et communautés amérindiennes situées plus au sud du Québec voudront obtenir des ententes négociées avec les gouvernements du Québec et du Canada. S'il y a actuellement cinq ententes signées avec les Cris, les Inuits et les Naskapis, il en reste encore plusieurs à signer avec les sept autres nations du Québec. Nos médias écrits et électroniques pourraient certes jouer un rôle important dans la diffusion de l'information sur les dossiers de négociations et la connaissance générale des Autochtones. Malheureusement, ils remplissent le plus souvent bien mal leur rôle en se contentant de coller à la nouvelle immédiate et de pratiquer du sensationnalisme sans analyse approfondie.

Parmi les journaux régionaux, seul Le Soleil a réalisé une bonne couverture de l'ensemble des débats de la commission par l'entremise de sa journaliste Monique Giguère. Quant au journal Le Quotidien de Saguenay (ancienne Chicoutimi), il a rapporté presque uniquement les interventions des personnes originaires de la région du Saguenay et celles des politiciens ou ex-politiciens comme Guy Chevrette. À ma connaissance, les débats ont aussi eu quelques échos dans de petits hebdomadaires régionaux comme Plein-Jour Côte-Nord et le Journal de la Haute-Côte-Nord, mais surtout à partir de certains mémoires dont ils ont obtenu copie. C'est ainsi que les accusations de " concurrence déloyale » se sont même retrouvées en page couverture des éditions du 31 janvier et du 14 février du journal Plein Jour. 


\section{Les interventions des parlementaires}

\section{$\underline{\text { Retour à la table des matières }}$}

Les audiences de la commission parlementaire se sont déroulées presque sans aucune manifestation d'esprit partisan, en dehors des reproches adressés au gouvernement du Parti Québécois pour le manque ou le retard dans la diffusion de l'information et le processus de consultation. Les trois partis et leurs représentants, le Parti québécois (PQ), le Parti libéral (PLQ) et l’Alliance démocratique du Québec (ADQ) se sont solidairement manifestés en faveur de l'entente de principe et l'ont même défendue face aux opposants. Les deux élus qui sont intervenus le plus souvent et le plus longuement furent le ministre des Affaires Autochtones d’alors, Rémy Trudel, et le représentant de l’opposition officielle Geoffrey Kelley. Si le premier s'est plusieurs fois perdu dans ses élans oratoires quelque peu ampoulés, il a quand même mené les débats de façon articulée et conséquente avec la politique du PQ qui prône le dialogue, le rapprochement et le partenariat avec les Autochtones du Québec comme en témoignent les ententes récentes avec les Cris et les Inuits. Le second a été plus efficace et souvent plus au point dans ses interventions et ses questions et il a démontré une excellente connaissance des différents dossiers concernant les Autochtones. Il aurait pu devenir un excellent ministre des Affaires autochtones dans le nouveau cabinet libéral ${ }^{1}$, mais cela ne s’est pas produit ainsi. L’ADQ était représenté par un seul député, François Corriveau, du comté de Saguenay, mais il avait si peu de temps pour intervenir à la toute fin de chacune des présentations (d'une durée limitée à 60 minutes) qu'il n’a jamais vraiment eu l'occasion de plonger dans le vif du sujet.

Il en est de même pour le reste des députés de la majorité ou de l’opposition qui ont souvent pratiqué la chaise musicale étant donné qu'à certains moments trois commissions parlementaires se tenaient en même temps. Je donnerais quand même une mention honorable à Christos Sirros ${ }^{2}$ qui a fait des interventions inté-

1 N.d.R. : Le Parti libéral a pris le pouvoir à la suite des élections du 14 avril 2003.

2 Ancien ministre libéral, C. Sirros représentait le parti libéral à la Commission des institutions. 
ressantes lorsqu'il était présent et qui a proposé pendant la séance de clôture que le parlement adopte l'entente de principe par une résolution lors de la reprise des travaux de la chambre le 11 mars. Malheureusement, le déclenchement des élections a eu la préséance dans l'ordre du jour politique du parti au pouvoir, le projet d'entente de principe n'a fait l'objet que d'une motion en faveur de la poursuite des négociations et ce, très tard en soirée, au grand dam des chefs des communautés innues concernées qui espéraient mieux. De fait, même si elle a été paraphée par les négociateurs en chef des trois partis, l'entente de principe n’a été entérinée officiellement ni par le gouvernement du Québec ni par celui du Canada. C’est pourquoi elle demeure fragile, d'où l'inquiétude des chefs qu'elle soit remise en question dans certains de ses éléments, comme la reconnaissance des droits territoriaux.

\section{Qui a peur des Innus?}

$\underline{\text { Retour à la table des matières }}$

En réponse à cette question on pourrait affirmer spontanément : surtout les gens de Saguenay et de Sept-Îles. En effet, c’est à ces deux endroits que les réactions négatives à l'entente ont été les plus vigoureuses et que se sont rapidement mis sur pied trois regroupements qui font campagne contre celle-ci avec de très forts appuis dans les médias locaux. Dans leurs interventions publiques les leaders ont fait preuve d'une simplification navrante des fondements de l'entente de principe et même d'une déformation vraisemblablement volontaire de ceux-ci pour ameuter le plus de personnes possible contre l'entente. Cette levée de bouclier locale est d'autant plus difficile à comprendre que la communauté innue de SeptÎles n'est même pas en négociation et qu'elle ne le sera pas de si tôt. Pourquoi donc de si nombreux citoyens de Sept-Îles se sentent-ils tant menacés ? Voilà une question fort complexe à laquelle il est difficile de répondre sans une étude sérieuse de la question qui nécessiterait une recherche plus approfondie. Je peux quand même avancer quelques explications de mon cru :

a) La communauté (officiellement « la bande ») innue de Uashat Mak ManiUtenam (ou Sept-Îles-Malioténam) a un effectif de plus de 3000 personnes habi- 
tantàdeux réserves, dont une enclavée dans la ville de Sept-Îles et une autre située à environà14 km à l'est. Le poids démographique de la communauté prend constamment de l'importance par rapport à celui des allochtones, parce que le taux de natalité est beaucoup plus élevé chez les Innus. Comme dans la plupart des autres communautés amérindiennes, la population innue locale est très jeune et les occasions de frictions entre gangs de jeunes sont plus fréquentes, comme cela a été relevé dans les médias au cours de l'automne 2002.

b) Récemment, la bande a réalisé de nombreux investissements dans le développement de ses infrastructures et du côté du logement. De même, elle est propriétaire du plus gros centre commercial local situé sur la réserve depuis de nombreuses années et elle est entrée en partenariat avec une importante chaîne de quincailleries qui s'y est installée depuis peu. Cette apparence extérieure d'une nouvelle richesse chez les Innus et cette volonté affichée de la bande d'accaparer une partie des activités commerciales locales semble déranger de nombreux SeptÎliens.

c) Le conseil de bande est à l'origine d'un mouvement de réoccupation des territoires de chasse qui s'est traduit, par exemple, par l'occupation du terrain de camping d'une ZEC, action qui a suscité beaucoup de remous et qui a aussi été rapportée par la télévision. Cette nouvelle affirmation concrète de l'existence de droits ancestraux et la référence très claire à la reconnaissance de ceux-ci par les derniers jugements de Cour ont mené directement au discours démagogique de certains leaders d'opinion affirmant que maintenant les Indiens (localement les Innus) ont tous les droits et qu'ils bénéficient de la complicité des gouvernements qui les privilégient au détriment de la majorité des « Blancs ».

d) Cette méfiance envers les gouvernements, en particulier celui du Québec, peut étonner dans une région reconnue comme un château fort péquiste depuis très longtemps. Vient-elle principalement de partisans de partis politiques de l'opposition? Je ne saurais le dire, mais il est symptomatique que le principal leader de la cabale contre l'entente de principe, André Forbes, se soit présenté comme candidat indépendant dans le comté de Duplessis dans le but avoué de damer le pion à la nouvelle candidate du PQ. L'entente avec les Innus, et les Innus eux-mêmes en fait, sont donc devenus un enjeu électoral local important. Cependant, l'élection de la candidate péquiste démontre que cet enjeu ne semble pas 
avoir influencé le vote de façon significative, le candidat Forbes n’ayant obtenu que 1334 votes, par ailleurs.

e) Finalement, sans parler directement de racisme, il est assez évident que la volonté de maintenir leur différence culturelle qu'affichent de plus en plus les Innus de Sept-Îles rencontre une opposition tout aussi marquée de la part d'une bonne partie de la population sept-îlienne. Comme l'attestent plusieurs indices, tels que le refus de laisser parler des personnes lors de séances publiques d'information et les lettres de menaces envoyées à certaines personnes appuyant l'entente, la situation a atteint un haut niveau d'intolérance. Est-ce un prélude à des affrontements racistes ? J'espère que non, mais le climat de tension sociale à Sept-Îles inquiète beaucoup.

La situation est quelque peu différente dans la ville de Saguenay et la tension n’y est pas aussi élevée, malgré les agitations et les abus de langage de son maire, Jean Tremblay, et du maître à penser des « disparitionnistes », Russel Bouchard. Ici encore on peut se demander pourquoi l'opposition est aussi virulente étant donné la grande distance qui sépare cette ville des territoires touchés par le projet d'entente de principe, soit l'Innu Assi et le Nitassinan de Mashteuiatsh. Comme les leaders politiques des principales entités administratives (municipalités, MRC) concernées directement par l'entente se sont prononcés en faveur de l'entente, comment se fait-il que l'éloignement attise l'opposition ? D’après certaines explications que l'on m’a données, des individus propriétaires d'entreprises, de pourvoiries et de baux de villégiature à l'intérieur du Nitassinan sentent leurs intérêts personnels menacés. Selon d'autres, des intérêts politiques partisans chez des membres influents du Parti libéral seraient derrière cette opposition étant donné que le dossier est devenu public pendant une période pré-électorale. On avance aussi que le mécontentement dû à la situation économique stagnante de la région et l'émigration importante des jeunes peuvent expliquer cette crispation. Finalement, le combat personnel contre la reconnaissance de quelques droits différents pour les Amérindiens en général et les Innus en particulier est au centre des motivations de l'historien disparitionniste local et de ses partisans.

Sur le plan strictement politique, les Innus sont donc victimes d'une conjoncture politique immédiate qui en ont fait des boucs émissaires des opposants au 
gouvernement du Parti québécois. Sur un autre plan, juridique et social celui-là, ils font face à une opposition plus limitée selon moi, mais tenace, qui sous l'argument de l'égalité de tous en droit ne veut leur reconnaître aucun droit particulier, surtout pas des droits aborigènes et encore moins territoriaux. Même si l'opposition des premiers s'estompe à la suite des élections du 14 avril, celle des seconds demeurera tenace et ils continueront à vouloir semer l'inquiétude et même la peur parmi leurs concitoyens.

\section{Références}

$\underline{\text { Retour à la table des matières }}$

ASH M., 1993, Home and Native Land : Aboriginal Rights and the Canadian Constitution. Vancouver, University of British Columbia Press.

ANONYME, 1994, Revendications territoriales globales des nations Atikamekw et Montagnaise : Offre du Gouvernement du Québec. s.l., s.é.

ANONYME, 2002, Entente de principe d'ordre général entre les Premières Nations de Mamuitun et de Nutashkuan et le gouvernement du Québec et le gouvernement du Canada. s.l., s.é.

BOUCHARD R., 1995, Le dernier des Montagnais de la préhistoire au début du XVIIIe siècle. Vie et mort de la nation Ilnu. Chicoutimi, s.é.

CANADA. AFFAIRES INDIENNES ET DU NORD, 1987, La politique des revendications territoriales globales. Ottawa, Approvisionnements et Services Canada.

CHAREST P., 1995, «Compte-rendu de Russel BOUCHARD : Le dernier des Montagnais de la préhistoire au début du XVIIIe siècle. Vie et mort de la nation Ilnu », Anthropologie et Sociétés, 19, 3 : 245-247.

—, 2003, Du nomadisme à l'approche commune : un projet social pour les Innus. Mémoire présenté devant la Commission des Institutions, Assemblée nationale du Québec, 12 février.

CHEVRETTE G., 2003, Pour la négociation d'un traité juste et équitable. s.l., s.é. 
CONSEIL ATTIKAMEK-MONTAGNAIS, 1979, « Nishastanan Nitasinan Notre terre, nous l'aimons et nous y tenons ", Recherches amérindiennes au Québec, IX, 3 : 171-182.

CONSEIL TRIBAL MAMUITUN, 1996, Proposition d'entente de principe en vue de la conclusion d'une entente finale entre d'une part le Conseil tribal Mamuitun et d'autre part le Gouvernement du Canada et le Gouvernement du Québec. s.l., s.é.

CUMMING P. et N. MICKENBERG, 1972, Native Rights in Canada. Toronto, Indian-Eskimo Association of Canada.

DAWSON N.-M., 2001, Feu, fourrures et foi déplacèrent les Montagnais : Histoire et destin de ces tribus nomades d'après les archives de l'époque coloniale. Sherbrooke, s.é.

KULSHINSKY P. (dir.), 1996, Unjust Relations : Aboriginal Rights in Canadian Courts. Toronto, Oxford University Press.

LAJOIE A. (dir.), 1996, Le statut juridique des peuples autochtones au Québec et le pluralisme. Cowansville, Éditions Yvon Blais.

LONG J. A., M. BOLDT et L. LITTLE BEAR (dir.), 1991, Aboriginal Rights : Toward an Understanding. Lethbridghe, Law Foundation.

MORSE B. (dir.), 1991, Aboriginal Peoples and the Law : Indian, Métis and Inuit Rights in Canada. Ottawa, Carleton University Press.

Paul Charest

Groupe d'études inuit et circumpolaires - GÉTIC

Université Laval

Québec (Québec)

G1K $7 P 4$

Canada 